\title{
Application of Multi-Objective PSO Algorithm for Power System Stability Enhancement by Means of SSSC
}

\author{
Ali Ajami and Mehdi Armaghan
}

\begin{abstract}
In this paper, on the basis of the theoretical analysis of a single-machine infinite-bus (SMIB), using its modified linearized Phillips-Heffron model installed with SSSC, the potential of the SSSC supplementary controller to enhance the dynamic stability of a power system is evaluated by measuring the electromechanical controllability through singular value decomposition (SVD) analysis. This controller is tuned to simultaneously shift the undamped electromechanical modes to a prescribed zone in the s-plane. The problem of robustly SSSC based damping controller is formulated as an optimization problem according to the eigenvalue-based multi-objective function comprising the damping factor, and the damping ratio of the undamped electromechanical modes to be solved using particle swarm optimization technique (PSO) that has a strong ability to find the most optimistic results. To ensure the robustness of the proposed damping controller, the design process takes into account a wide range of operating conditions. The effectiveness of the proposed controller is demonstrated through controllability measure, eigenvalue analysis, nonlinear time-domain simulation and some performance indices studies. The results analysis reveals that the tuned PSO based SSSC controller using the proposed multi-objective function has an excellent capability in damping power system low frequency oscillations and enhance greatly the dynamic stability of the power systems.
\end{abstract}

Index Terms-Power system dynamic stability, Power transmission control, particle swarm optimization, static synchronous series compensator.

\section{INTRODUCTION}

When large power systems are interconnected by relatively weak tie lines, low frequency oscillations are observed. These oscillations may sustain and grow to cause system separation if no adequate damping is available [1]. In order to damp these power system oscillations and increase system oscillations stability, the installation of power system stabilizer is both economical and effective. PSSs have been used for many years to add damping to electromechanical oscillations. However, PSSs suffer a drawback of being liable to cause great variations in the voltage profile and they may even result in leading power factor operation and losing system stability under severe disturbances, especially those

Manuscript received Mar 5, 2010.

Ali Ajami is with the electrical engineering department of Azarbaijan university of Tarbiat Moallem, Tabriz, Iran, phone: +98-914-3183362; fax: +98-412-4327548; e-mail: ajami@azaruniv.edu

Mehdi Armaghan is M. Sc. student of electrical engineering department of Azarbaijan university of Tarbiat Moallem, Tabriz, Iran, Mehdi_63.armaghan@yahoo.com three phase faults which may occur at the generator terminals [2]. In recent years, the fast progress in the field of power electronics had opened new opportunities for the application of the FACTS devices as one of the most effective ways to improve power system operation controllability and power transfer limits [3, 4]. FACTS devices can cause a substantial increase in power transfer limits during steady state via controlling of magnitude and phase angle of bus voltages and transmission line reactance. Because of the extremely fast control action associated with FACTS device operations, they have been very promising candidates for utilization in power system damping enhancement. It has been observed that utilizing a feedback supplementary control, in addition to the FACTS device primary control, can considerably improve system damping and can also improve system voltage profile, which is advantageous over PSSs. Static Synchronous Series Compensator (SSSC) is one of the important members of FACTS family which can be installed in series in the transmission lines. With the capability to change its reactance characteristic from capacitive to inductive, the SSSC is very effective in controlling power flow in power systems [5]. An auxiliary stabilizing signal can also be superimposed on the power flow control function of the SSSC so as to improve power system oscillation stability [6]. The applications of SSSC for power oscillation damping, stability enhancement and frequency stabilization can be found in several references [5-8]. The influence of compensation degree and operation mode of SSSC on small disturbance and transient stability is also reported in the literature [9-11]. Most of these proposals are based on small disturbance analysis that required linearization of the system involved. However, linear methods cannot properly capture complex dynamics of the system, especially during major disturbances. This presents difficulties for tuning the FACTS controllers since the controllers tuned to provide desired performance at small signal condition do not guarantee the acceptable performance in large disturbances. A conventional lead/lag controller structure is preferred by the power system utilities because of the easy online tuning and also lack of assurance of the stability by some adaptive or variable structure techniques. The problem of FACTS controller parameter tuning is a complex exercise. A number of conventional techniques have been reported in the literature pertaining to design problems of conventional power system stabilizers namely: the eigenvalue assignment, mathematical programming, gradient procedure for optimization and also the modern control theory. 
Unfortunately, the conventional techniques are time consuming as they are iterative and require heavy computation burden and slow convergence. In addition, the search process is susceptible to be trapped in local minima and the solution obtained may not be optimal [12].

In this paper, PSO technique is used for the optimal tuning of SSSC based damping controller in order to enhance the damping of power system low frequency oscillations and achieves the desired level of robust performance under different operating conditions. PSO is a novel population based metaheuristic, which utilize the swarm intelligence generated by the cooperation and competition between the particle in a swarm and has emerged as a useful tool for engineering optimization. Unlike the other heuristic techniques, it has a flexible and well balanced mechanism to enhance the global and local exploration abilities. Also, it suffices to specify the objective function and to place finite bounds on the optimized parameters. This algorithm has also been found to be robust in solving problems featuring nonlinearity, non differentiability and high dimensionality [13-17].

In this study, singular value decomposition (SVD) is used to select the control signal which is most suitable for damping the electromechanical (EM) mode oscillations. This is done as SVD analysis can be readily used to evaluate the EM mode controllability of the SSSC controller. A SMIB system equipped with a SSSC controller is used in this study. The problem of robust SSSC based damping controller design is formulated as a multiobjective optimization problem. The multiobjective problem is concocted to optimize a composite set of two eigenvalue based objective functions comprising the desired damping factor, and the desired damping ratio of the lightly damped and undamped electromechanical modes. The controller is automatically tuned with optimization an eigenvalue based multi objective function by PSO to simultaneously shift the lightly damped and undamped electromechanical modes to a prescribed zone in the s-plane such that the relative stability is guaranteed and the time domain specifications concurrently secured. The effectiveness of the proposed controller is demonstrated through controllability measure, eigenvalue analysis, nonlinear time simulation studies and some performance indices to damp low frequency oscillations under different operating conditions. Results evaluation show that the proposed multiobjective function based tuned damping controller achieves good robust performance for a wide range of operating conditions and is superior to both designed controller using the single objective functions.

\section{PARTICLE SWARM OptIMIZATION}

\section{A. Overview}

A novel population based optimization approach, called Particle Swarm Optimization (PSO) approach, was introduced first in [13]. This new approach has many advantages; it is simple, fast and can be coded in few lines. Also, its storage requirement is minimal.

Moreover, this approach is advantageous over evolutionary and genetic algorithms in many ways [4]. First, PSO has memory. That is, every particle remembers its best solution (local best) as well as the group best solution (global best).

Another advantage of PSO is that the initial population of the PSO is maintained, and so there is no need for applying operators to the population, a process that is time and memory storage consuming. In addition, PSO is based on "constructive cooperation", between particles, in contrast with the genetic algorithms, which are based on "the survival of the fittest',

PSO starts with a population of random solutions " particles" in a D-dimension space. The ith particle is represented by $\mathrm{Xi}=(\mathrm{xi} 1, \mathrm{xi} 2, \ldots, \mathrm{xiD})$. Each particle keeps track of its coordinates in hyperspace, which are associated with the fittest solution it has achieved so far. The value of the fitness for particle $\mathrm{i}$ (pbest) is also stored as $\mathrm{Pi}=$ (pi1, pi2, .., piD). The global version of the PSO keeps track of the overall best value (gbest), and its location, obtained thus far by any particle in the population $[13,14]$.

PSO consists of, at each step, changing the velocity of each particle toward its pbest and gbest according to (1). The velocity of particle $\mathrm{i}$ is represented as $\mathrm{Vi}=(\mathrm{vi} 1, \mathrm{vi} 2, \ldots, \mathrm{viD})$. Acceleration is weighted by a random term, with separate random numbers being generated for acceleration toward pbest and gbest. The position of the ith particle is then updated according to (2) $[13,14]$ :

$$
\begin{aligned}
v_{i d}= & w^{*} v_{i d}+c_{1} * \operatorname{rand}() *\left(p_{i d}-x_{i d}\right) \\
& +c_{2} * \operatorname{rand}() *\left(p_{g d}-x_{i d}\right) \\
x_{i d}= & x_{i d}+v_{i d}
\end{aligned}
$$

Where, pid $=$ pbest and pgd $=$ gbest. Several modifications have been proposed in the literature to improve the PSO algorithm speed and convergence toward the global minimum. One modification is to introduce a local-oriented paradigm (lbest) with different neighborhoods. It is concluded that gbest version performs best in terms of median number of iterations to converge. However, pbest version with neighborhoods of two is most resistant to local minima. PSO algorithm is further improved via using a time decreasing inertia weight, which leads to a reduction in the number of iterations [17].

\section{B. PSO algorithm [4]}

1) Initialize an array of particles with random positions and their associated velocities to satisfy the inequality constraints.

2) Check for the satisfaction of the equality constraints and modify the solution if required.

3) Evaluate the fitness function of each particle.

4) Compare the current value of the fitness function with the Compare the current value of the fitness function with the value is less, then assign the current fitness value to pbest and assign the current coordinates (positions) to pbestx.

5) Determine the current global minimum fitness value among the current positions.

6) Compare the current global minimum with the previous global minimum (gbest). If the current global minimum is better than gbest, then assign the current global minimum to gbest and assign the current coordinates (positions) to gbestx.

7) Change the velocities according to (1). 
8) Move each particle to the new position according to (2) and return to Step 2.

9) Repeat Step 2-8 until a stopping criterion is satisfied or the maximum number of iterations is reached.

\section{POWER SYSTEM MODEL}

A single-machine infinite-bus (SMIB) power system installed with SSSC is investigated, as shown in Fig. 1 [7]. The SSSC consists of a boosting transformer with a leakage reactance $x_{S C T}$, a three-phase GTO based voltage source converter (VSC), and a DC capacitor $\left(C_{D C}\right)$. The two input control signals to the SSSC are $m$ and $\psi$. Signal $m$ is the amplitude modulation ratio of the pulse width modulation (PWM) based VSC. Also, signal $\psi$ is the phase of the injected voltage and is kept in quadrature with the line current (inverter losses are ignored). Therefore, the compensation level of the SSSC can be controlled dynamically by changing the magnitude of the injected voltage. Hence, if the SSSC is equipped with a damping controller, it can be effective in improving power system dynamic stability.

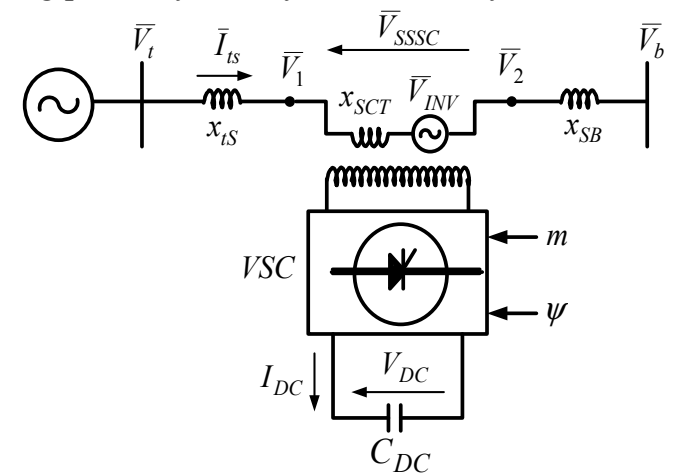

Fig. 1. Single-machine infinite-bus power system with SSSC.

\section{A. Power system nonlinear model with SSSC}

The dynamic model of the SSSC is required in order to study the effect of the SSSC for enhancing the small signal stability of the power system. The system data is given in the Appendix. By applying Park's transformation and neglecting the resistance and transients of the transformer, the SSSC can be modeled as [7]:

$$
\begin{aligned}
& \bar{I}_{t s}=I_{t s d}+j I_{t s q}=I_{T S} \angle \varphi \\
& \bar{V}_{I N V}=m k V_{D C}(\cos \psi+j \sin \psi)=m k V_{D C} \angle \psi \\
& \psi=\varphi \pm 90^{\circ} \\
& \dot{V}_{D C}=\frac{d V_{D C}}{d t}=\frac{I_{D C}}{C_{D C}} \\
& \dot{V}_{D C}=\frac{m k}{C_{D C}}\left(I_{t s d} \cos \psi+I_{t s q} \sin \psi\right)
\end{aligned}
$$

Where, $k$ is the ratio between $\mathrm{AC}$ and DC voltages and is dependent on the inverter structure.

The nonlinear dynamic model of the power system of Fig. 1 is [7]:

$$
\begin{gathered}
\dot{\delta}=\omega_{b} \omega \\
\dot{\omega}=\left(P_{m}-P_{e}-D \omega\right) / M \\
\dot{E}_{q}^{\prime}=\left(-E_{q}+E_{f d}\right) / T_{d o}^{\prime}
\end{gathered}
$$

$$
\dot{E}_{f d}=-\frac{1}{T_{A}} E_{f d}+\frac{K_{A}}{T_{A}}\left(V_{t o}-V_{t}\right)
$$

Where,

$$
\begin{gathered}
P_{e}=E_{q}^{\prime} I_{t s q}+\left(x_{q}-x_{d}^{\prime}\right) I_{t s d} I_{t s q}, E_{q}=E_{q}^{\prime}+\left(x_{d}-x_{d}^{\prime}\right) I_{t s d}, \\
V_{t}=\sqrt{\left(E_{q}^{\prime}-x_{d}^{\prime} I_{t s d}\right)^{2}+\left(x_{q} I_{t s q}\right)^{2}} .
\end{gathered}
$$

\section{B. Power system linearized model}

A linear dynamic model is obtained by linearizing the nonlinear model around an operating condition. By linearizing (3)-(10) we can obtain:

$$
\begin{gathered}
\Delta \dot{\delta}=\omega_{b} \Delta \omega \\
\Delta \dot{\omega}=\left(-\Delta P_{e}-D \Delta \omega\right) / M \\
\Delta \dot{E}_{q}^{\prime}=\left(-\Delta E_{q}+\Delta E_{f d}\right) / T_{d o}^{\prime} \\
\Delta \dot{E}_{f d}=-\frac{1}{T_{A}} \Delta E_{f d}-\frac{K_{A}}{T_{A}} \Delta V_{t} \\
\Delta \dot{V}_{D C}=K_{7} \Delta \delta+K_{8} \Delta E_{q}^{\prime}+K_{9} \Delta V_{D C}+K_{d m} \Delta m+K_{d \psi} \Delta \psi
\end{gathered}
$$

Where,

$$
\begin{aligned}
& \Delta P_{e}=K_{1} \Delta \delta+K_{2} \Delta E_{q}^{\prime}+K_{p D C} \Delta V_{D C}+K_{p m} \Delta m+K_{p \psi} \Delta \psi \\
& \Delta E_{q}=K_{4} \Delta \delta+K_{3} \Delta E_{q}^{\prime}+K_{q D C} \Delta V_{D C}+K_{q m} \Delta m+K_{q \psi} \Delta \psi \\
& \Delta V_{t}=K_{5} \Delta \delta+K_{6} \Delta E_{q}^{\prime}+K_{v D C} \Delta V_{D C}+K_{v m} \Delta m+K_{v \psi} \Delta \psi .
\end{aligned}
$$

$K_{1}, K_{2}, \ldots, K_{9}, K_{p u}, K_{q u}, K_{d u}$ and $K_{v u}$ are linearization constants and are dependent on system parameters and the operating condition.

The state space model of power system is given by:

$$
\dot{x}=A x+B u
$$

Where, the state vector $x$, control vector $u, A$ and $B$ are:

$$
\begin{aligned}
& x=\left[\begin{array}{lllll}
\Delta \delta & \Delta \omega & \Delta E_{q}^{\prime} & \Delta E_{f d} & \Delta V_{D C}
\end{array}\right]^{T} \\
& u=\left[\begin{array}{ll}
\Delta m & \Delta \psi
\end{array}\right]^{T} \\
& A=\left[\begin{array}{ccccc}
0 & \omega_{b} & 0 & 0 & 0 \\
-\frac{K_{1}}{M} & -\frac{D}{M} & -\frac{K_{2}}{M} & 0 & -\frac{K_{p D C}}{M} \\
-\frac{K_{4}}{T_{d o}^{\prime}} & 0 & -\frac{K_{3}}{T_{d o}^{\prime}} & \frac{1}{T_{d o}^{\prime}} & -\frac{K_{q D C}}{T_{d o}^{\prime}} \\
-\frac{K_{A} K_{5}}{T_{A}} & 0 & -\frac{K_{A} K_{6}}{T_{A}} & -\frac{1}{T_{A}} & -\frac{K_{A} K_{v D C}}{T_{A}} \\
K_{7} & 0 & K_{8} & 0 & K_{9}
\end{array}\right] \\
& B=\left[\begin{array}{cc}
0 & 0 \\
-\frac{K_{p m}}{M} & -\frac{K_{p \psi}}{M} \\
-\frac{K_{q m}}{T_{d o}^{\prime}} & -\frac{K_{q \psi}}{T_{d o}^{\prime}} \\
-\frac{K_{A} K_{v m}}{T_{A}} & -\frac{K_{A} K_{v \psi}}{T_{A}} \\
K_{d m} & K_{d \psi}
\end{array}\right]
\end{aligned}
$$

The block diagram of the linearized dynamic model of the SMIB power system with SSSC is shown in Fig. 2.

\section{SSSC based damping controller}

The SSSC damping controller structure is shown in Fig. 3, where $u$ can be $m$ or $\psi$. It comprises gain block, signal washout block and lead-lag compensator [3]. 


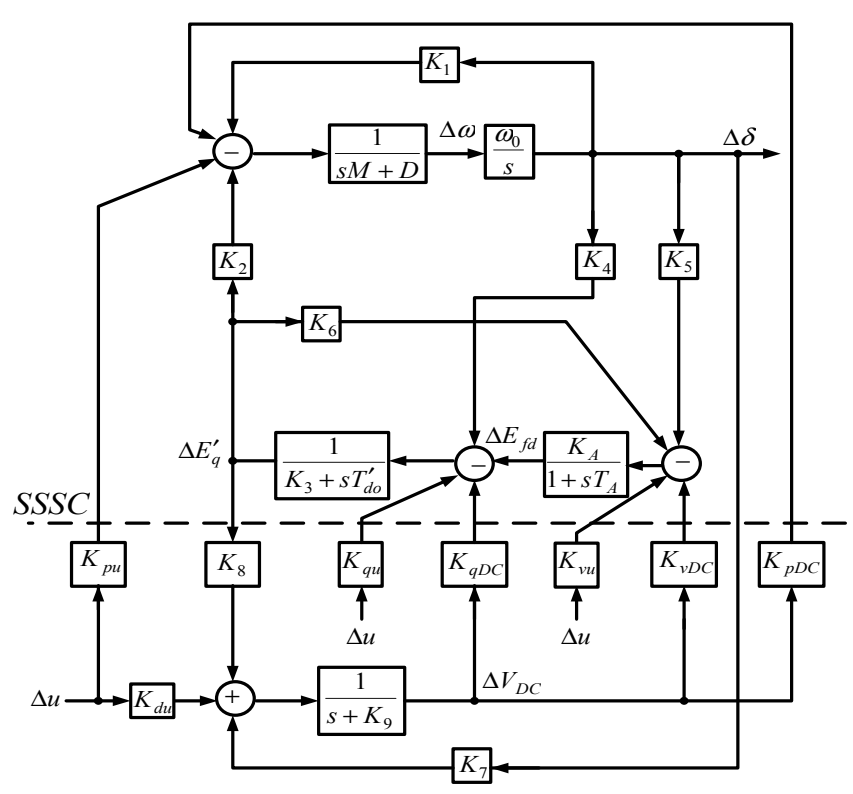

Fig. 2. Modified Phillips-Heffron model of a SMIB system with SSSC.

\section{Optimization Problem}

In the proposed method, we must tune the SSSC controller parameters optimally to improve overall system dynamic stability in a robust way under different operating conditions. For our optimization problem, an eigenvalue based multi-objective function reflecting the combination of damping factor and damping ratio is considered as follows $[18,19]$ :

$$
J_{3}=J_{1}+a J_{2}
$$

Where, $J_{1}=\sum_{\sigma_{i} \geq \sigma_{0}}\left(\sigma_{0}-\sigma_{i}\right)^{2}, J_{2}=\sum_{\zeta_{i} \leq \zeta_{0}}\left(\zeta_{0}-\zeta_{i}\right)^{2}, \sigma_{i}$ and $\zeta_{i}$ are the real part and the damping ratio of the $i$ th eigenvalue, respectively. The value of $a$ is chosen at 10 . The value of $\sigma_{0}$ determines the relative stability in terms of damping factor margin provided for constraining the placement of eigenvalues during the process of optimization. The closed loop eigenvalues are placed in the region to the left of dashed line as shown in Fig. 4a, if only $J_{1}$ were to be taken as the objective function. Similarly, if only $J_{2}$ is considered, then it limits the maximum overshoot of the eigenvalues as shown in Fig. 4b. In the case of $J_{2}, \zeta_{0}$ is the desired minimum damping ratio which is to be achieved. When optimized with $\mathrm{J}_{3}$, the eigenvalues are restricted within a D-shaped area as shown shaded in Fig. 4c.

The optimization problem can be stated as:

Minimize $J_{i}$

Subject to

$$
\begin{aligned}
& K^{\text {min }} \leq K \leq K^{\text {max }} \\
& T_{1}^{\text {min }} \leq T_{1} \leq T_{1}^{\text {max }} \\
& T_{2}^{{ }^{\text {min }}} \leq T_{2} \leq T_{2}^{\text {max }} \\
& T_{3}^{{ }^{\text {min }}} \leq T_{3} \leq T_{3}^{\text {max }} \\
& T_{4}{ }^{\text {min }} \leq T_{4} \leq T_{4}^{\text {max }}
\end{aligned}
$$

Typical ranges of the optimized parameters are [0.01-100] for $K$ and [0.01-1] for $T_{1}, T_{2}, T_{3}$ and $T_{4}$.

Tuning a controller parameter can be viewed as an optimization problem in multi-modal space as many settings of the controller could be yielding good performance.
Traditional method of tuning doesn't guarantee optimal parameters and in most cases the tuned parameters needs improvement through trial and error. In PSO based method, the tuning process is associated with an optimality concept through the defined objective function and the time domain simulation. The designer has the freedom to explicitly specify the required performance objectives in terms of time domain bounds on the closed loop responses. Hence the PSO methods yield optimal parameters and the method is free from the curse of local optimality. In view of the above, the proposed approach employs PSO to solve this optimization problem and search for optimal set of SSSC-based damping controller parameters.

In this study, the values of $\sigma_{0}$ and $\zeta_{0}$ are taken as -1.5 and 0.2 , respectively. In order to acquire better performance, number of particle, particle size, number of iteration, $c_{1}$ and $c_{2}$ is chosen as 30, 5, 50, 2 and 2, respectively. Also, the inertia weight, $w$, is linearly decreasing from 0.9 to 0.4 .

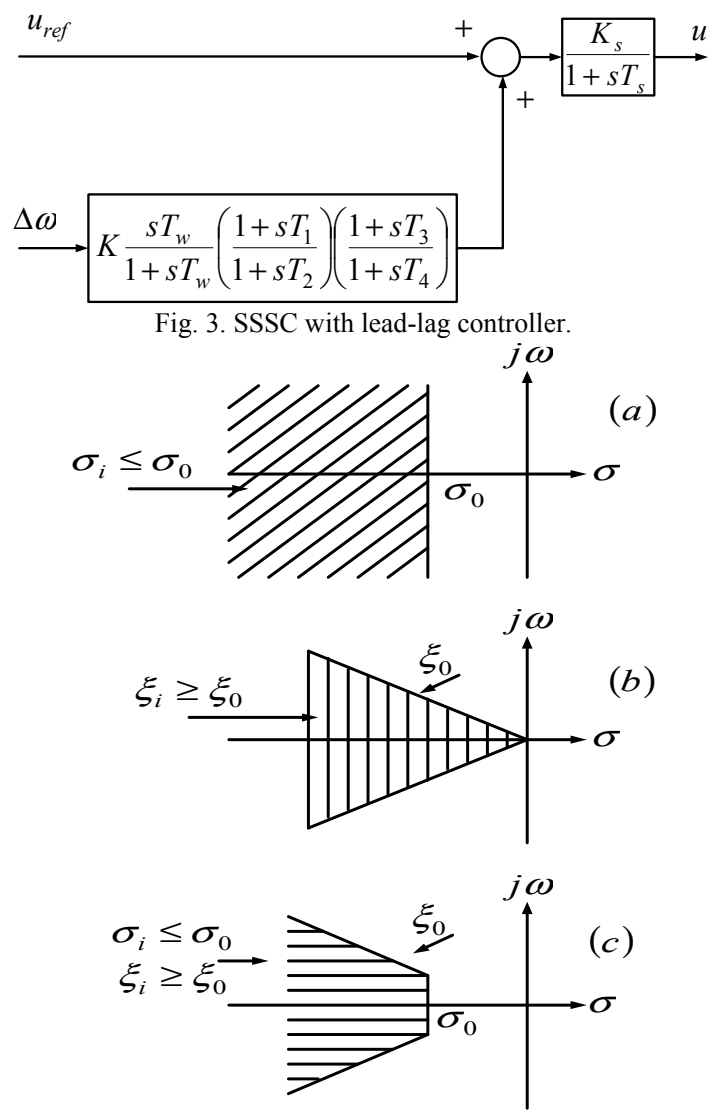

Fig.4. Region of eigenvalue location for objective functions.

\section{Controllability Measure}

In this paper, for measuring the controllability of electromechanical mode by a given input, the singular value decomposition (SVD) is employed. Mathematically, if G is an $\mathrm{m} \times \mathrm{n}$ complex matrix then there exist unitary matrices $\mathrm{W}$ and $\mathrm{V}$ with dimensions of $\mathrm{m} \times \mathrm{m}$ and $\mathrm{n} \mathrm{x} \mathrm{n}$ respectively such that $\mathrm{G}$ can be written as:

Where,

$$
G=W \Sigma V^{H}
$$

$$
\Sigma=\left[\begin{array}{cc}
\Sigma_{1} & 0 \\
0 & 0
\end{array}\right], \Sigma_{1}=\operatorname{diag}\left(\sigma_{1}, \ldots, \sigma_{r}\right)
$$


With $\sigma_{1} \geq \ldots \geq \sigma_{r} \geq 0 \quad$ where $r=\min \quad\{\mathrm{m}, \mathrm{n}\} \quad$ and $\sigma_{1}, \ldots, \sigma_{r}$ are the singular values of $G$.

The minimum singular value $\sigma_{r}$ represents the distance of the matrix $G$ from the all matrices with a rank of $r-1$. This property can be utilized to quantify modal controllability [20]. In this study, the matrix $B$ in (16) can be written as $B=\left[b_{1}, b_{2}\right]$ where $b_{i}$ is the column of matrix $B$ corresponding to the $i$ th input. The minimum singular value of the matrix $\left[\lambda I-A: b_{i}\right]$ indicates the capability of the $i$ th input to control the mode associated with the eigenvalue $\lambda$. As a matter of fact, the higher minimum singular value, the higher controllability of this mode by the input considered. Having been identified, the controllability of the electromechanical mode can be examined with all inputs in order to identify the most effective one to control that mode.

\section{V.Simulation RESULTS}

To assess the effectiveness of the proposed stabilizer, three different loading conditions given in Table 1 were considered. At each loading condition the SSSC $\psi$ and $m$ parameters have been recalculated so that SSSC has no injections to the system.

TABLE1. LOADING CONDITION

\begin{tabular}{|l|l|l|}
\hline Loading & $P(\mathrm{pu})$ & $Q(\mathrm{pu})$ \\
\hline Nominal & 1 & 0.45 \\
\hline Light & 0.65 & 0.35 \\
\hline Heavy & 1.4 & 0.6 \\
\hline
\end{tabular}

\section{A. Controllability measure}

SVD is employed to measure the controllability of the EM mode from each of the two inputs: $m$ and $\psi$. The minimum singular value, $\sigma_{\min }$, is estimated over a wide range of operating conditions. For SVD analysis, $P_{e}$ ranges from 0.05 to $1.4 \mathrm{pu}$ and $Q_{e}=[-0.4,0,0.4]$. At each loading condition, the system model is linearized, the EM mode is identified, and the SVD-based controllability measure is implemented.

For comparison purposes, the minimum singular value for each of the $t w o$ inputs at $Q_{e}=-0.4,0.0$ and 0.4 pu is shown in Fig. 5. From this figure, it can be noticed that EM mode controllability via $\psi$ in different loading conditions is always higher than $m$.

\section{B. Eigenvalues Analysis}

The PSO algorithm has been applied to search for the optimal parameter settings of the supplementary controller so that the objective functions are optimized. It should be noted that PSO algorithm is run several times and then optimal set of SSSC controller parameters is selected. The final values of the optimized parameters with both single objective functions $J_{1}, J_{2}$ and the multi-objective function $J_{3}$ in the nominal loading condition are given in Table 2 .

The electromechanical modes and the damping ratios obtained for all operating conditions both with and without $\psi$-based controller in the system are given in Table 3.
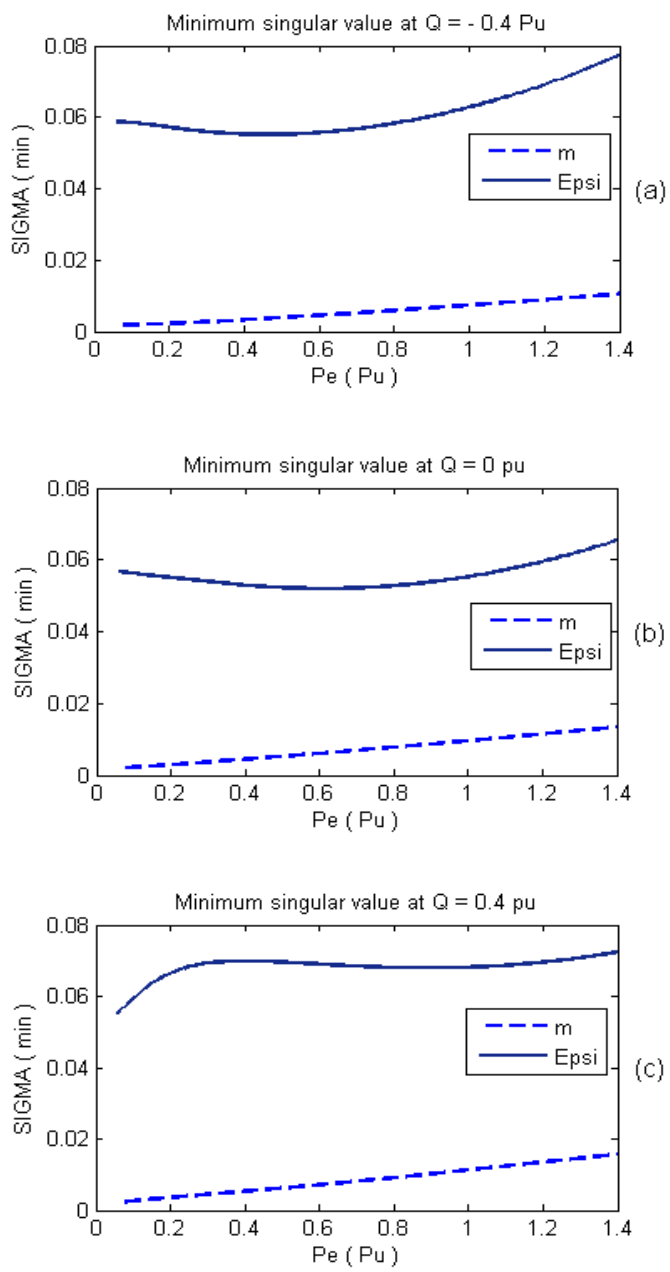

Fig. 5. Minimum singular value at different loading (a) $Q_{e}=-0.4 \mathrm{pu}$, (b) $Q_{e}=0 \mathrm{pu}$, (c) $Q_{e}=0.4 \mathrm{pu}$.

\section{Non Linear Time Domain Simulation}

The single-machine infinite-bus system shown in Fig. 1 is considered for nonlinear simulation studies. 6-cycle 3-phase fault at $\mathrm{t}=1 \mathrm{~s}$, on the infinite bus is occurred, at all loading conditions given in Table 1, to study the performance of the proposed controller. The performance of the controller when the multi-objective function is used in the design is compared to that of the controllers designed using the single objective functions $J_{1}$ and $J_{2}$. The speed deviation, internal voltage deviation, DC voltage deviation and electrical power deviation based on the $\psi$ controller in three different loading conditions are shown in Figs. 6, 7 and 8. It can be seen that the PSO based SSSC controller tuned using the multiobjective function achieves good robust performance, provides superior damping in comparison with the other objective functions and enhance greatly the dynamic stability of power systems.

TABLE2. THE OPTIMAL PARAMETER SETTINGS OF PROPOSED CONTROLLER BASED ON $\Psi$ FOR DIFFERENT OBJECTIVE FUNCTION.

\begin{tabular}{|l|l|l|l|}
\hline Controller parameters & $J_{1}$ & $J_{2}$ & $J_{3}$ \\
\hline$K$ & 100 & 70.22 & 41.95 \\
\hline$T_{1}$ & 0.99 & 0.59 & 0.97 \\
\hline$T_{2}$ & 0.011 & 0.051 & 0.045 \\
\hline$T_{3}$ & 0.117 & 0.847 & 1 \\
\hline$T_{4}$ & 0.184 & 0.455 & 0.625 \\
\hline
\end{tabular}


TABLE3. EIGENVALUES AND DAMPING RATIOS OF ELECTROMECHANICAL MODES WITH AND WITHOUT $\Psi$ CONTROLLER.

\begin{tabular}{|l|l|l|l|}
\hline Objective functions & $\begin{array}{l}\text { Nominal loading condition } \\
\text { Eigenvalue (damping ratio) }\end{array}$ & $\begin{array}{l}\text { Light loading condition } \\
\text { Eigenvalue (damping ratio) }\end{array}$ & $\begin{array}{l}\text { Heavy loading condition } \\
\text { Eigenvalue (damping ratio) }\end{array}$ \\
\hline Without controller & $-9.3048 \pm 13.4343 \mathrm{i},(0.5694)$ & $-9.8281 \pm 15.3165 \mathrm{i},(0.5401)$ & $-8.0836 \pm 9.7787 \mathrm{i},(0.6371)$ \\
& $0.0631 \pm 4.8279 \mathrm{i},(-0.013)$ & $0.1311 \pm 4.7758 \mathrm{i},(-0.027)$ & $-0.6170 \pm 5.2721 \mathrm{i},(0.1162)$ \\
& -1.6285 & -0.9150 & -2.2896 \\
\hline$J_{1}$ & $-1.5 \pm 5.88 \mathrm{i},(0.247)$ & $-1.5 \pm 8 \mathrm{i},(0.1841)$ & $-1.59 \pm 6.106 \mathrm{i},(0.2519)$ \\
& $-1.61 \pm 8.63 \mathrm{i},(0.183)$ & $-1.5 \pm 5.24 \mathrm{i},(0.2742)$ & $-3.053 \pm 7.64 \mathrm{i},(0.3711)$ \\
& $-42.02 \pm 26.83 \mathrm{i},(0.842)$ & $-16.05 \pm 19.04 \mathrm{i},(0.6445)$ & $-96.29 \pm 14.58 \mathrm{i},(0.9887)$ \\
& $-1.62,-6.53$ & $-1.54,-2.77$ & $-1.96,-2.29$ \\
& -147.13 & -109.85 & -35.7 \\
\hline$J_{2}$ & $-1.15 \pm 5.62 \mathrm{i},(0.2)$ & $-1.76 \pm 5.34 \mathrm{i},(0.3126)$ & $-1.098 \pm 5.38 \mathrm{i},(0.2)$ \\
& $-1.67 \pm 8.16 \mathrm{i},(0.2)$ & $-1.62 \pm 7.38 \mathrm{i},(0.2138)$ & $-3.91 \pm 9.19 \mathrm{i},(0.3921)$ \\
& $-22.55 \pm 21.98 \mathrm{i},(0.716)$ & $-15.7 \pm 18.49 \mathrm{i},(0.6472)$ & $-97.31 \pm 12.47 \mathrm{i},(0.9919)$ \\
& $-1.63,-2.18$ & $-1.32,-3.33$ & $-2.28,-4.24$ \\
& -107.07 & -108.86 & -32.97 \\
\hline$J_{3}$ & $-1.86 \pm 4.07 \mathrm{i},(0.416)$ & $-1.53 \pm 4.75 \mathrm{i},(0.3065)$ & $-1.5 \pm 0.0325 \mathrm{i},(0.9998)$ \\
& $-2.14 \pm 10.37 \mathrm{i},(0.202)$ & $-5.5 \pm 8.66 \mathrm{i},(0.5361)$ & $-1.98 \pm 5.25 \mathrm{i},(0.3537)$ \\
& $-23.83 \pm 22.25 \mathrm{i},(0.73)$ & $-15.65 \pm 16.21 \mathrm{i},(0.6945)$ & $-8.63 \pm 9.23 \mathrm{i},(0.6827)$ \\
& $-1.61,-1.72$ & $-1.7,-1.51$ & $-2.19,-18.78$ \\
& -107.26 & -105.57 & -99.97 \\
\hline
\end{tabular}
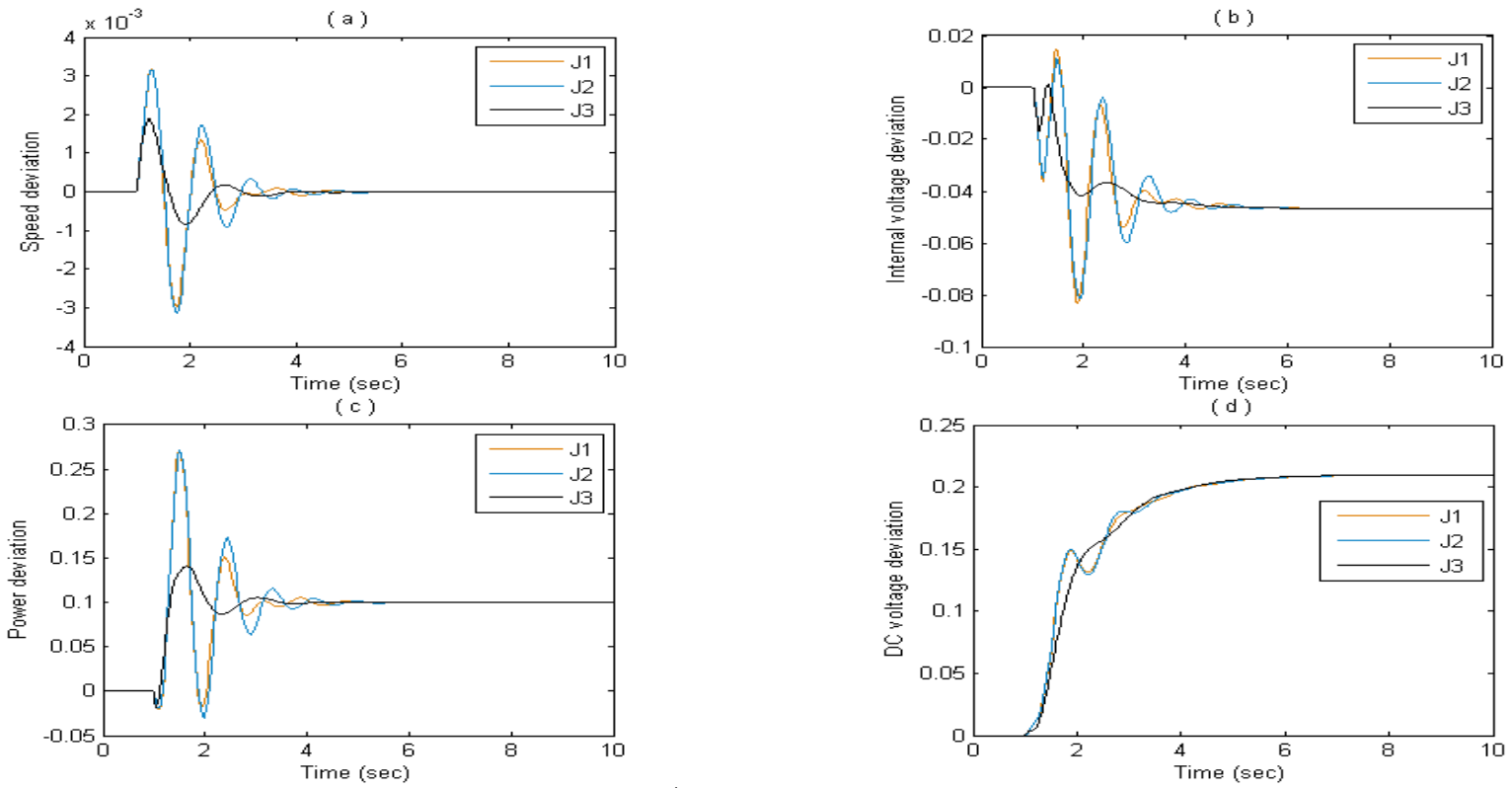

Fig. 6. Dynamic responses for (a) $\Delta \omega$, (b) $\Delta E_{q}^{\prime}$, (c) $\Delta P_{e}$, (d) $\Delta V_{D C}$ whit $\psi$ controller at light loading condition.
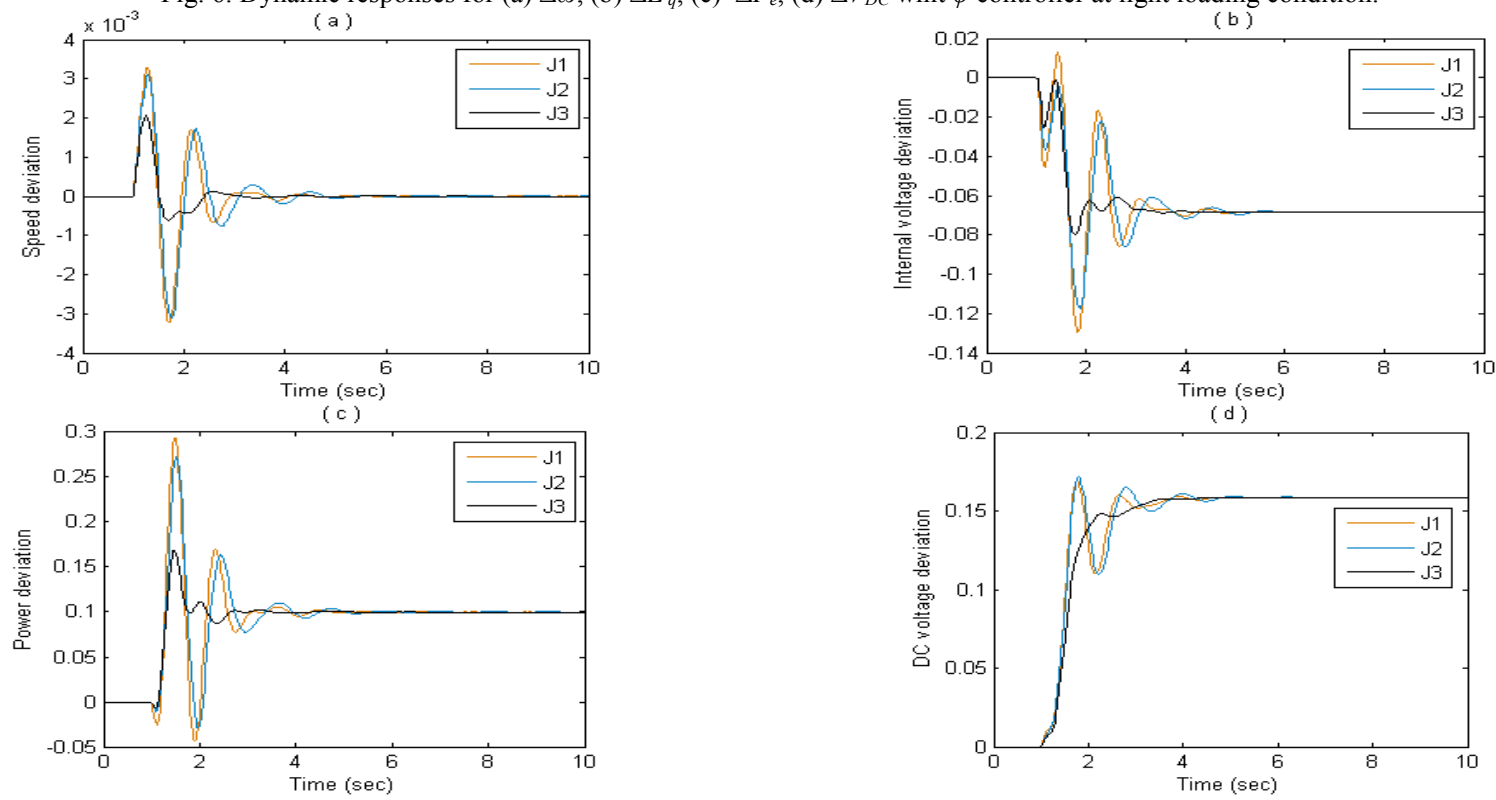

Fig. 7. Dynamic responses for (a) $\Delta \omega$, (b) $\Delta E_{q}^{\prime}$, (c) $\Delta P_{e}$, (d) $\Delta V_{D C}$ whit $\psi$ controller at nominal loading condition. 

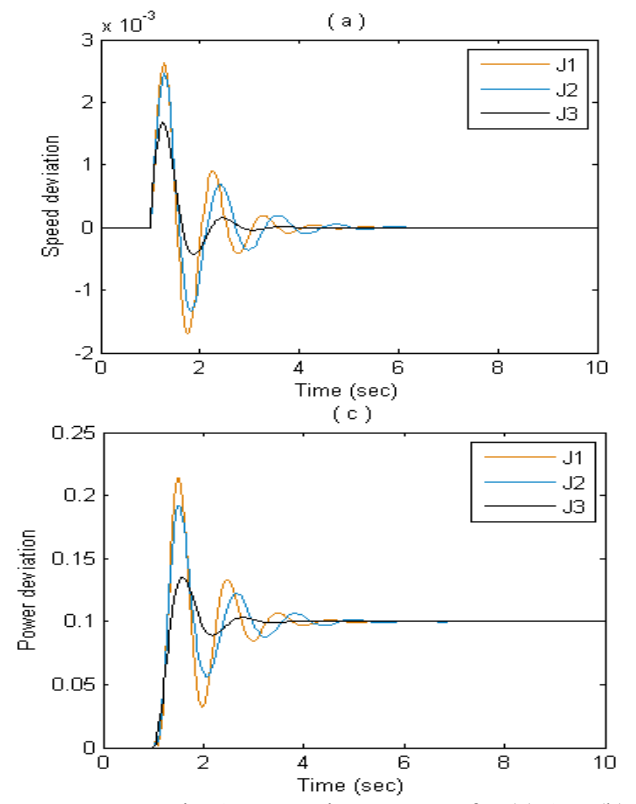

Fig. 8. Dynamic responses for (a) $\Delta \omega$, (b) $\Delta E_{q}^{\prime}$, (c) $\Delta P_{e}$, (d) $\Delta V_{D C}$ whit $\psi$ controller at heavy loading condition.

\section{CONCLUSIONS}

In this paper, SVD has been employed to evaluate the EM mode controllability to the two SSSC control signals. It has been shown that EM mode controllability via $\psi$ in different loading conditions is always higher than $m$. The stabilizer is tuned to simultaneously shift the undamped electromechanical modes of the machine to a prescribed zone in the s-plane. A multi-objective problem is formulated to optimize a composite set of objective functions comprising the damping factor, and the damping ratio of the undamped electromechanical modes. The design problem of the controller is converted into an optimization problem which is solved by a PSO technique with the eigenvalue based multi-objective function. The effectiveness of the proposed SSSC controller for improving transient stability performance of a power system is demonstrated by a weakly connected example power system subjected to different disturbances. The eigenvalue analysis and nonlinear time domain simulation results show the effectiveness of the proposed controller using multi-objective function and its ability to provide good damping of low frequency oscillations.

\section{APPENDIX}

The test system parameters are:

Machine:

$D=0 ; x_{d}=1 ; x_{q}=0.6 ; x_{d}^{\prime}=0.3 ; M=8 ; T_{d o}^{\prime}=5.044 ; f=60 ; V_{t}=1$;

Exitation system: $K_{A}=120 ; T_{A}=0.05$;

Transmission line: $x_{t S}=0.35 ; x_{S B}=0.6$;

SSSC:

$C_{D C}=0.25 ; V_{D C}=1 ; K_{s}=1.2 ; T_{s}=0.05 ; T_{w}=0.01 ; x_{S C T}=0.15$.

\section{REFERENCES}

[1] P. Kundur, "Power system stability and control", McGraw-Hill, 1994.

[2] Keri AJF, Lombard X, Edris AA, "Unified power flow controller: modeling and analysis", IEEE Transaction on Power Delivery, 1999, 14(2):648-54.

[3] H. Shayeghi, H.A. Shayanfar, S. Jalilzadeh, A. Safari, "A PSO based unified power flow controller for damping of power system
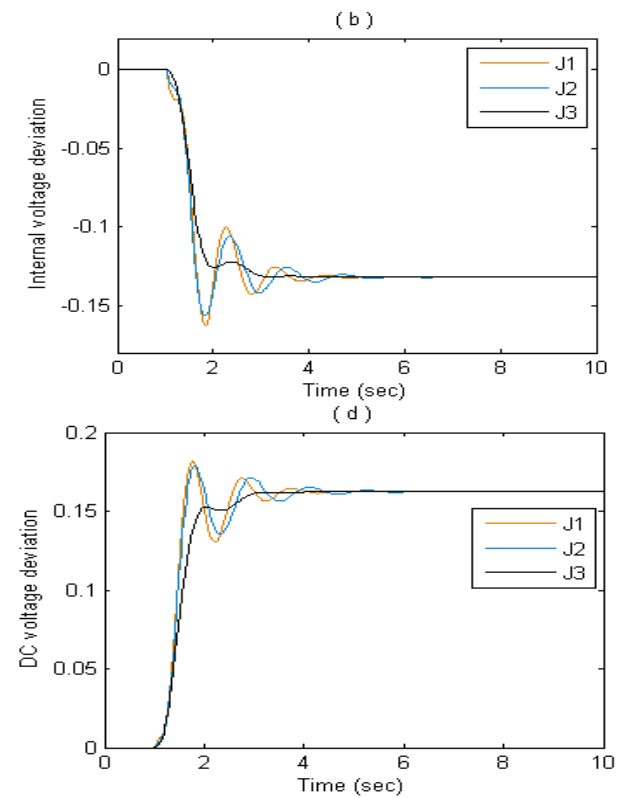

oscillations", Energy Conversion and Management 2009, 50: 25832592.

[4] Al-Awami AT, Abdel-Magid YL, Abido MA, "A particle swarmbased approach of power system stability enhancement with unified power flow controller", Elect Power Energy Syst 2007,29:251-9.

[5] L. Gyugyi, C. D. Schauder, K. K. Sen, "Static synchronous series compensator: a solid-state approach to the series compensation of transmission lines", IEEE Transaction on Power Delivery, vol. 12, no.1, pp. 406-17, Jan. 1997.

[6] H. F. Wang, "Static synchronous series compensator to damp power system oscillations", Electric Power Systems Research, vol. 54, pp. 113-119, 2000.

[7] Wang. H. F, "Design of SSSC damping controller to improve power system oscillation stability", AFRICON 1999, IEEE, 1, 495 -500.

[8] Hingorani NG, Gyugyi L, "Understanding FACTS: concepts and technology of flexible AC transmission systems", Wiley-IEEE Press, 1999.

[9] F. A. R. Al Jowder, B. T. Ooi, "Series Compensation of Radial Power System by a Combination of SSSC and Dielectric Capacitors", IEEE Transactions on Power Delivery, vol. 20, no. 1, pp.,pp458-465, January 2005.

[10] M.S. Castro, H.M. Ayres, V.F. da Costa, L.C.P. da Silva, "Impacts of the SSSC control modes on small-signal transient stability of power system", Electric Power Systems Research, vol. 77, pp. 1-9, 2007.

[11] F. A. R. Al Jowder, "Influence of Mode of Operation of the SSSC on the Small Disturbance and Transient Stability of a Radial Power System", IEEE Transactions onPower Systems, vol. 20, no. 2, pp935942, May 2005.

[12] Y.L.Abdel-Magid, M.A.Abido, "Robust coordinated design of excitation and TCSC-based stabilizers using genetic algorithms", International Journal of Electrical Power \& Energy Systems, vol. 69, no. 2-3, pp. 129-141. 2004.

[13] Kennedy J, Eberhart R, "Particle swarm optimization", Proc IEEE Int Conf Neural Networks 1995,4:1942-8.

[14] Eberhart R, Kennedy J, "A new optimizer using particle swarm theory", In: Proc Sixth Int Symp Micro Machine Human Science, vol. 4-6 October, 1995, p. 39-43.

[15] Kennedy J, Eberhart R, Shi Y, "Swarm intelligence", San Francisco, Morgan Kaufmann Publishers, 2001.

[16] Z.L.Gaing, "A particle swarm optimization approach for optimum design of PID controller in AVR system", IEEE Transaction on Energy Conversions, vol. 9, no. 2, pp. 384-391, 2004.

[17] Clerc M, Kennedy J, "The particle swarm-explosion, stability, and convergence in a multidimensional complex space", IEEE Trans Evolut Comput 2002,6(1): 58-73.

[18] Abdel-Magid YL, Abido MA, "Optimal multiobjective design of robust power system stabilizers using genetic algorithms", IEEE Trans Power Syst 2003,18(3):1125-32.

[19] M. A. Abido, Y. L. Abdel-Magid, "Optimal design of power system stabilizers using evolutionary programming", IEEE Trans. on Energy Conversion, Vol. 17, No. 4, 2002, pp. 429-436. 
[20] A. M. A. Hamdan, "An Investigation of the Significance of Singular Value Decomposition in Power System Dynamics", Int. Journal of Electrical Power and Energy Systems, Vol. 21, 1999, pp. 417-424.

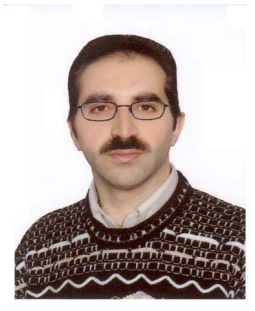

Ali Ajami received his B.Sc. and M. Sc. degrees from the Electrical and Computer Engineering Faculty of Tabriz University, Iran, in Electronic Engineering and Power Engineering in 1996 and 1999, respectively, and his Ph.D. degree in 2005 from the Electrical and Computer Engineering Faculty of Tabriz University, Iran, in Power Engineering. His main research interests are dynamic and steady state modeling and analysis of FACTS devices, harmonics and power quality compensation systems, microprocessors, DSP and computer based control systems.

Mehdi Armaghan received his B.Sc. degree from Islamic Azad University in electrical engineering. He is M. Sc. student of electrical engineering department of Azarbaijan University of Tarbiat Moallem from 2009. His main research interests are dynamic and steady state modeling and analysis of FACTS devices, harmonics and power quality compensation systems. 